\title{
Association of IL17RC and COL6A1 genetic polymorphisms with susceptibility to ossification of the thoracic posterior longitudinal ligament in Chinese patients
}

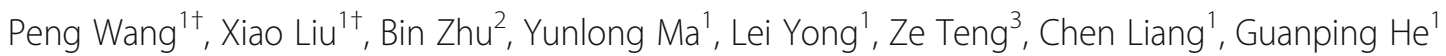
and Xiaoguang Liu ${ }^{1 *}$ (D)

\begin{abstract}
Background: In our previous whole-genome sequencing study of 30 unrelated northern Chinese Han patients, we identified six single nucleotide polymorphisms (SNPs) in the interleukin 17 receptor C (IL17RC) and collagen type VI a1 chain (COL6A1) genes that were potentially associated with thoracic ossification of the posterior longitudinal ligament (T-OPLL). To determine whether these six SNPs are associated with susceptibility to T-OPLL in the northern Chinese Han population, we performed a case-control association study to confirm specific susceptible loci in the expanded samples.
\end{abstract}

Methods: The six SNPs in the IL17RC and COL6A1 genes were analyzed in 200 northern Chinese individuals (100 patients and 100 control subjects) using the Sequenom system.

Results: The genotype distributions and allele frequencies of each SNP in the control and patient groups were compared. rs201153092, rs13051496, rs199772854, rs76999397, and rs189013166 showed potential pathogenic loci for T-OPLL in the northern Chinese Han population, whereas rs151158105 did not. At the genotype level, the differences in the genotype frequencies of rs201153092, rs13051496, rs199772854, rs76999397, and rs189013166 between T-OPLL cases and controls reached statistical significance.

Conclusions: To the best of our knowledge, this is the first association study of susceptibility genes in Han Chinese patients with T-OPLL. The results revealed five SNPs in the IL17RC and COL6A1 genes that represented potentially pathogenic mutations in patients with T-OPLL.

Keywords: Thoracic, Ossification of the posterior longitudinal ligament, LL17RC, COL6A1, Association study

\section{Background}

Ossification of the posterior longitudinal ligament (OPLL), which involves pathological heterotopic ossification of this region, can lead to symptoms of spinal cord compression and radiculopathy in affected patients. The disease has a marked ethnic predilection, with epidemiological studies suggesting that OPLL is found almost exclusively in Japanese, Chinese, and Korean individuals [1]. Approximately $70 \%$ of

\footnotetext{
*Correspondence: xglius@vip.sina.com

${ }^{\dagger}$ Equal contributors

${ }^{1}$ Department of Orthopedics, Peking University Third Hospital, 49 North Garden Street, Haidian District, Beijing 100191, People's Republic of China

Full list of author information is available at the end of the article
}

OPLL cases affect the cervical spine, whereas $15 \%$ occur in the thoracic spine [2]. Ohtsuka et al. reported that the prevalence of thoracic OPLL (T-OPLL) in Japanese individuals is $0.8 \%$, which is lower than that of cervical OPLL (3.2\%) [3]. Although the most common site of OPLL is the cervical spine, T-OPLL is considered more severe.

As T-OPLL is slowly progressive, the majority of patients develop symptoms only when the disease progresses to an advanced stage, when severe spinal cord injury occurs. Clinical manifestations include sensory disturbances, dyskinesia, fecal and urinary incontinence, and even paraplegia. The disability rate in T-OPLL is much higher than that in cervical OPLL. Currently, 
treatment approaches for T-OPLL are limited, and surgery is the only effective treatment. However, owing to the unusual blood supply and anatomical structure associated with this disease, surgery for T-OPLL is complicated; it is difficult to avoid surgical complications, and the risk of paralysis is extremely high. The optimal surgical methods for T-OPLL, as well as the timing of the surgery, remain controversial, thus it remains a challenge for clinicians to perform timely and effective treatment of the disease [4].

Genetics are considered to be a predominant factor in the etiology of OPLL. Several East Asian groups have performed candidate gene linkage and association studies, which have suggested that a number of polymorphisms in osteogenic genes are associated with the occurrence and development of cervical OPLL. To date, 16 genes/loci that are linked to OPLL susceptibility have been reported [5], including toll-like receptor 5 (TLR5) [6], collagen type XII a2 chain (COL11A2) [7], COL6A1 [8-10], runt-related transcription factor 2 (RUNX2) [11], bone morphogenetic protein 2 (BMP2) [12], bone morphogenetic protein 4 (BMP4) [13], transforming growth factor $\beta 1$ (TGFB1) [14], interleukin $1 \beta(I L 1 B)$ [15], and interleukin 15 receptor subunit $\alpha(I L 15 R A)$ [16]. However, these findings have not been sufficiently reproducible, and usually only one SNP was examined to evaluate the gene, and thus there is a lack of linkage disequilibrium (LD) studies on multiple single nucleotide polymorphisms (SNPs) [17]. In addition, no genetic studies have assessed the causes of T-OPLL.

In our previous whole-genome sequencing (WGS) study of 30 unrelated northern Chinese Han patients, four different algorithms [including SIFT(http://sift.jcvi.org/) [18], PolyPhen-2 (http://genetics.bwh.harvard.edu/pph2/) [19], MutationTaster (http://www.mutationtaster.org/) [20], and GERP++ (http://mendel.stanford.edu/SidowLab/ downloads/gerp/) [21]] were used to predict deleterious variations at different SNP loci. We found that rs201153092 was predicted to be deleterious by four algorithms, rs199772854 was predicted by three algorithms, and rs151158105, rs13051496, rs76999397, and rs189013166 were predicted by two algorithms (Table 1). In the present study, we aimed to identify genetic factors for T-OPLL by performing a case-control study of six SNPs in two genes (COL6A1 and IL17RC) potentially associated with susceptibility to T-OPLL in the northern Chinese Han population.

\section{Methods}

\section{Case selection and grouping}

The study protocol was approved by the Ethics Committee for Human Subjects of the Peking University Third Hospital. Informed consent was provided by all participating individuals. A total of 100 unrelated northern Chinese Han T-OPLL patients with myelopathy and/or neurological dysfunction [ 47 men (mean age, $51.00 \pm 3.30$ years) and 53 women (mean age, $52.34 \pm 6.09$ years)] and 100 sex-matched unrelated healthy controls [45 men (mean age, $55.84 \pm 3.06$ years) and 55 women (mean age, $54.36 \pm$ 5.69 years)] were enrolled in this study between January 2010 and October 2016. Diagnosis of T-OPLL was performed by specialists based on clinical symptoms and radiological examinations (including computed tomography and magnetic resonance imaging) of the thoracic spine. The appearance of OPLL observed on the radiographs was classified into four subtypes: (i) segmental, (ii) continuous, (iii) mixed, and (iv) local [22]. Individuals who had lumbar spondylolisthesis, ankylosing spondylitis, diffuse idiopathic skeletal hyperostosis, and disc herniation of the thoracic spine were included in the study, and none were taking any drugs known to affect bone or calcium metabolism.

\section{SNP selection and genotyping}

EDTA-anticoagulated peripheral blood samples were obtained from all participants for DNA extraction. Genomic DNA samples were extracted from peripheral leukocytes with the standard procedure using a Wizard Genomic DNA Purification Kit (Promega Corporation, Madison, WI, USA). Six SNPs distributed among the two genes were selected based on findings from our previous WGS studies. The polymerase chain reaction (PCR) fragments were submitted for Sanger sequencing at the Beijing Genomics Institute, and the forward and reverse sequence reads were assembled and analyzed in DNAStar version

Table 1 Mutation findings for 30 patients with T-OPLL by WGS

\begin{tabular}{|c|c|c|c|c|c|c|c|c|c|}
\hline Gene & SNP ID & Chromosome & Nucleotide change & Protein change & 1000G (EAS) & SIFT & PP2 & MutationTaster & GERR++ \\
\hline COL6A1 & rs201153092 & 21 & c. $1534 G>A$ & p.Gly512Ser & 0 & $\mathrm{D}$ & $\mathrm{D}$ & D & $\mathrm{R}$ \\
\hline COL6A1 & rs13051496 & 21 & $c .2669 C>T$ & p.Ser890Leu & 0 & T & B & $D$ & $R$ \\
\hline COL6A1 & rs151158105 & 21 & c. $1298 \mathrm{G}>\mathrm{A}$ & p.Arg433Gln & 0 & $\mathrm{D}$ & B & N & $\mathrm{R}$ \\
\hline IL17RC & rs199772854 & 3 & c. $2275 \mathrm{C}>\mathrm{A}$ & p.Leu759lle & 0 & $\mathrm{D}$ & $\mathrm{D}$ & N & $\mathrm{R}$ \\
\hline IL17RC & rs76999397 & 3 & c. $1908 \mathrm{G}>\mathrm{A}$ & p.Ala636Ala & 0.00378 & $\mathrm{D}$ & B & N & R \\
\hline IL17RC & rs189013166 & 3 & c. $2238 \mathrm{G}>\mathrm{A}$ & p.Gly746Gly & 0 & $\mathrm{D}$ & B & N & $\mathrm{R}$ \\
\hline
\end{tabular}

$1000 \mathrm{G}$ (EAS), 1000 Genomes (Asian); SIFT ( $D$ deleterious, $T$ tolerated); PP2, Polyphen-2 ( $D$ probably damaging, $P$ possibly damaging, $B$ benign); MutationTaster ( $D$ disease causing, $N$ polymorphism); GERP++ ( $R$ rejected substitutions, $S$ substitutions) 
7.1. Details of the six studied SNPs and the primer sequences are listed in Table 2. The six primers were used for PCR as described previously [23]. PCR was performed with 20 ng genomic DNA per $15-\mu$ r reaction mixture, containing $0.2 \mu \mathrm{M}$ of each primer, $200 \mu \mathrm{M}$ of deoxyribonucleotides, $50 \mathrm{mM} \mathrm{KCl}, 10 \mathrm{mM}$ Tris $\mathrm{HCl}(\mathrm{pH} 8.3), 1.5 \mathrm{mM}$ $\mathrm{MgCl}_{2}$, and 0.5 units of Taq DNA polymerase in a DNA Gradient PCR machine (Bio-Rad Laboratories, Inc., Hercules, CA, USA). The thermocycling conditions were as follows: initial denaturation at $95{ }^{\circ} \mathrm{C}$ for $10 \mathrm{~min}$; followed by 35 cycles of $95{ }^{\circ} \mathrm{C}$ for $30 \mathrm{~s}$, annealing at an assay-specific temperature $\left(48{ }^{\circ} \mathrm{C}\right.$ to $\left.65{ }^{\circ} \mathrm{C}\right)$ for $45 \mathrm{~s}$, and elongation at $72{ }^{\circ} \mathrm{C}$ for $45 \mathrm{~s}$; and a final terminal elongation step at $72{ }^{\circ} \mathrm{C}$ for $5 \mathrm{~min}$. The PCR products were analyzed by direct sequencing using a BigDye Terminator v3.1 Cycle Sequencing Kit (Thermo Fisher Scientific, Inc., Waltham, MA, USA) with POP- $7^{\text {ma }}$ Polymer in a 3730XL DNA Analyzer with Sequencing Analysis Software version 5.2 (Thermo Fisher Scientific, Inc., Waltham, MA, USA).

\section{Statistical analysis}

All statistical analyses were performed using SPSS v17.0 software (SPSS, Inc., Chicago, IL, USA). The $\chi^{2}$ test was used to determine whether individual variants were in equilibrium at each locus in the population (HardyWeinberg equilibrium), as well as the genotypic and allelic distributions. Pairwise LD was calculated as $\mathrm{D}^{\prime}$ and $R^{2}$ using Haploview version 4.2. The Pearson's $\chi^{2}$ test was used to determine the correlation between the variants and the disease based on the odds ratios (ORs) with 95\% confidence intervals (95\% CI). All statistical tests were two-sided, and significance was set at $P<0.05$.

\section{Results}

Six SNPs were genotyped among northern Chinese Han subjects. With the exception of rs151158105, which was incompatible with the Hardy-Weinberg equilibrium $(P=0.005)$, the remaining five SNPs were in HardyWeinberg equilibrium $(P>0.05)$.
The allelic frequencies and genotype distributions of the six studied SNPs are shown in Tables 3 and 4 . rs201153092, rs13051496, rs199772854, rs76999397, and rs189013166 showed significant associations with TOPLL $(P<0.05)$. The A allele frequency of rs201153092 was higher in T-OPLL cases (8.5\%) than in controls (0.5\%). The $\mathrm{T}$ allele frequency of rs13051496 was higher in T-OPLL cases $(5.5 \%)$ than in controls (1.0\%). The A allele frequency of rs199772854 was higher in T-OPLL cases (6\%) than in controls (1.0\%). The A allele frequency of rs76999397 was higher in T-OPLL cases (4.5\%) than in controls $(1.0 \%)$. The A allele frequency of rs189013166 was higher in T-OPLL cases $(4.0 \%)$ than in controls (0.5\%).

At the genotype level, the differences in the genotype frequencies at rs201153092, rs13051496, rs199772854, rs76999397, and rs189013166 reached statistical significance between T-OPLL cases and controls (recessive model; $P=0.00007707, P=0.009838, P=0.005582$, $P=0.02992$, and $P=0.01696$, respectively). No significant difference was observed in the genotype or allele frequencies of rs151158105 between T-OPLL cases and controls. The LD coefficients among rs201153092, rs151158105, and rs13051496 ranged from 0.91 to 1 , and the $R^{2}$ value ranged from 0.05 to 0.6 (Table 5). The LD coefficients among rs199772854, rs76999397, and rs189013166 were 1, and the $R^{2}$ value ranged from 0.63 to 0.81 (Table 6).

\section{Discussion}

OPLL is a condition that is characterized by the calcification of the soft tissues that connect the bones of the spine. Although the exact underlying cause is currently unknown, it has been suggested that it is a multifactorial condition that is influenced by several different genetic and environmental factors [17]. The accumulation of deleterious missense mutations in individual human genomes has been proposed to form the genetic basis of complex diseases [24], and WGS is considered to be a promising approach for the study of such diseases [5]. In

Table 2 Details of the six SNPS in COL6A1 and IL17RC and their associated primers

\begin{tabular}{|c|c|c|c|}
\hline Gene & SNP ID & Nucleotide substitution (M/m) & Primer sequence \\
\hline COL6A1 & rs201153092 & $\mathrm{G} / \mathrm{A}$ & $\begin{array}{l}\text { Forward 5'-TGAAAGGGTGAGTGTCCAA-3' } \\
\text { Reverse 5'-GTGCCCAGTCCACTAAAGAG-3' }\end{array}$ \\
\hline COL6A1 & rs13051496 & $\mathrm{C} / \mathrm{T}$ & $\begin{array}{l}\text { Forward 5'-AGCCACAACTITGACACCA-3' } \\
\text { Reverse 5'-GAAGCGGGTCACATAGCC-3' }\end{array}$ \\
\hline COL6A1 & rs151158105 & $\mathrm{G} / \mathrm{A}$ & $\begin{array}{l}\text { Forward 5'-CCTCCTGCCCAAGACA-3' } \\
\text { Reverse 5'-TCCAAAGAAGAACCCAAGC-3' }\end{array}$ \\
\hline ILITRC & rs199772854 & $\mathrm{C} / \mathrm{A}$ & $\begin{array}{l}\text { Forward 5'-CCCAACTGCCAGACTTCCT-3' } \\
\text { Reverse 5'-GCCACAGCCTGCGTAAAA-3' }\end{array}$ \\
\hline IL17RC & rs76999397 & $\mathrm{G} / \mathrm{A}$ & $\begin{array}{l}\text { Forward 5'-GGCTCTGCTCCTCTACTCAG-3' } \\
\text { Reverse 5'-AATGACGTTTGCCAGCCC-3' }\end{array}$ \\
\hline ILITRC & rs189013166 & $\mathrm{G} / \mathrm{A}$ & $\begin{array}{l}\text { Forward 5'-GGCTCTGCTCCTCTACTCAG-3' } \\
\text { Reverse 5'-AATGACGTTTGCCAGCCC-3' }\end{array}$ \\
\hline
\end{tabular}


Table 3 Allelic frequencies of the six SNPS

\begin{tabular}{|c|c|c|c|c|c|}
\hline \multirow[t]{2}{*}{ SNP ID } & \multirow[t]{2}{*}{$N$} & \multicolumn{2}{|c|}{ Allele frequency (\%) } & \multicolumn{2}{|c|}{ Versus control } \\
\hline & & Major allele & Minor allele & $P$ value $^{a}$ & Odds ratio $(95 \% \mathrm{Cl})$ \\
\hline rs201153092 & & $G$ & $A$ & & \\
\hline Cases & 100 & 91.50 & 8.50 & 0.000114 & $18.49(2.436-140.3)$ \\
\hline Controls & 100 & 99.50 & 0.50 & & \\
\hline rs13051496 & & C & $\mathrm{T}$ & & \\
\hline Cases & 100 & 94.50 & 5.50 & 0.01116 & $5.762(1.26-26.34)$ \\
\hline Controls & 100 & 99.00 & 1.00 & & \\
\hline rs151158105 & & G & $A$ & & \\
\hline Cases & 100 & 99.50 & 0.50 & 0.3167 & - \\
\hline Controls & 100 & 100.00 & 0.00 & & \\
\hline rs199772854 & & $C$ & A & & \\
\hline Cases & 100 & 94.00 & 6.00 & 0.006515 & $6.319(1.396-28.61)$ \\
\hline Controls & 100 & 99.00 & 1.00 & & \\
\hline rs76999397 & & G & A & & \\
\hline Cases & 100 & 95.50 & 4.50 & 0.03234 & $4.665(0.9951-21.87)$ \\
\hline Controls & 100 & 99.00 & 1.00 & & \\
\hline rs189013166 & & G & $A$ & & \\
\hline Cases & 100 & 96.00 & 4.00 & 0.01827 & $8.292(1.027-66.92)$ \\
\hline Controls & 100 & 99.50 & 0.50 & & \\
\hline
\end{tabular}

$N$ number of subjects

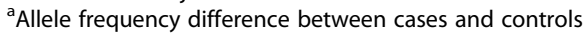

the present study, we performed association studies to confirm specific susceptibility loci in expanded samples.

In this study, rs201153092 and rs13051496 in COL6A1 were potential pathogenic loci for T-OPLL in the northern Chinese Han population. According to our data, the A allele frequency of rs201153092 was higher in T-OPLL cases than controls, which implied that the A allele could be a risk factor for the genetic susceptibility to T-OPLL and that the "GA" genotype enhanced the probability of the occurrence of T-OPLL. The T allele frequency of rs13051496 was higher in T-OPLL cases than in controls, which implied that the $\mathrm{T}$ allele could be a risk factor for the genetic susceptibility to T-OPLL and that the "CT" genotype enhanced the probability of T-OPLL susceptibility.

COL6A1, which encodes the $\alpha 1$ chain of type VI collagen, is located on chromosome 21q22.3 and spans approximately $23.3 \mathrm{kbp}$. COL6A1 encodes an extracellular matrix protein that may serve as a scaffold for osteoblastic or preosteoblastic cells or chondrocytes that subsequently undergo membranous or endochondral ossification [25]. Although the functional impacts of rs201153092 and rs13051496 in COL6A1 in bone metabolism remain elusive, the molecular variants of these extracellular proteins may be implicated in the ectopic bone formation observed in T-OPLL patients [26].

rs199772854, rs76999397, and rs189013166 in IL17RC were potential pathogenic loci for T-OPLL in the northern
Chinese Han population. The current study showed that the A allele frequency of rs199772854 was higher in TOPLL cases than in controls, which implied that the A allele could be a risk factor for the genetic susceptibility to T-OPLL and that the "CA" genotype enhanced the probability of T-OPLL susceptibility. The A allele frequency of rs76999397 was higher in T-OPLL cases than in controls, which implied that the A allele could be a risk factor for the genetic susceptibility to T-OPLL and that the 'GA' genotype was enhance the probability of T-OPLL susceptibility. In addition, the A allele frequency of rs189013166 was higher in T-OPLL cases than controls, which implied that the A allele could be a risk factor for the genetic susceptibility to T-OPLL and that the 'GA' genotype was enhance the probability of T-OPLL susceptibility.

The IL17RC gene encodes a single-pass type I transmembrane protein located between the chromosomal regions 3 p25.3 and 3p24.1, spanning approximately $16.55 \mathrm{kbp}$ [27]. IL17RC has been implicated as an important regulator of bone metabolism that accelerates osteoblast differentiation. A recent study indicated that the IL17RC protein plays an indispensable role in osteoblastogenesis [28]. Moreover, the TGF- $\beta$ signaling pathway is known to be associated with the initiation of ectopic ossification [29]. The IL17RC gene may also be involved in bone metabolism through canonical TGF- $\beta$ signaling. This study provides a basis for the study of the effects of these SNPs on OPLL in the future. 
Table 4 Genotype frequencies of the six SNPs

\begin{tabular}{|c|c|c|c|c|c|c|}
\hline \multirow[t]{2}{*}{ SNP ID } & \multirow[t]{2}{*}{$N$} & \multicolumn{3}{|c|}{ Genotype frequency (\%) } & \multicolumn{2}{|c|}{ Versus control } \\
\hline & & $\overline{\mathrm{MM}}$ & $\mathrm{Mm}$ & $\mathrm{mm}$ & $P$ value $^{a}$ & $P$ value ${ }^{b}$ \\
\hline rs201153092 & & GG & GA & $\mathrm{AA}$ & & \\
\hline Cases & 100 & 83 & 17 & 0 & - & 0.00007707 \\
\hline Controls & 100 & 99 & 1 & 0 & & \\
\hline rs13051496 & & CC & CT & $\pi$ & & \\
\hline Cases & 100 & 89 & 11 & 0 & - & 0.009838 \\
\hline Controls & 100 & 98 & 2 & 0 & & \\
\hline rs151158105 & & GG & GA & $\mathrm{AA}$ & & \\
\hline Cases & 100 & 99 & 1 & 0 & - & 0.3161 \\
\hline Controls & 100 & 100 & 0 & 0 & & \\
\hline rs199772854 & & CC & CA & $\mathrm{AA}$ & & \\
\hline Cases & 100 & 88 & 12 & 0 & - & 0.005582 \\
\hline Controls & 100 & 98 & 2 & 0 & & \\
\hline rs76999397 & & GG & GA & $\mathrm{AA}$ & & \\
\hline Cases & 100 & 90.91 & 9.09 & 0 & - & 0.02992 \\
\hline Controls & 100 & 98 & 2 & 0 & & \\
\hline rs189013166 & & GG & GA & $\mathrm{AA}$ & & \\
\hline Cases & 100 & 92 & 8 & 0 & - & 0.01696 \\
\hline Controls & 100 & 99 & 1 & 0 & & \\
\hline
\end{tabular}

$N$ number of subjects

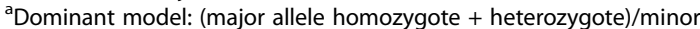
allele homozygote

${ }^{\mathrm{b}}$ Recessive model: major allele homozygote/(minor allele homozygote

+ heterozygote)

There were some limitations in the present study. First, the power of bioinformatics analyses and damaging-variant prediction algorithms are limited. The results of allelic frequencies and genotype distributions showed that there is no homogeneity of the disease-related allele and homozygous allies of pathological related minor allies in any of the T-OPLL patients, suggesting that the T-OPLL disease has highly genetic heterogeneities and complex pathogenesis. In addition, we lacked studies of co-efficiency between the allies of these two genes. To accomplish these issues, further genetic and functional studies, including studies with more participants of other ethnicities, are needed to confirm these positive findings.

\section{Conclusions}

To the best of our knowledge, this is the first association study of T-OPLL susceptibility genes in Han Chinese

Table 5 Linkage disequilibrium statistics among IL17RC SNPS $\left(\mathrm{D}^{\prime} / R^{2}\right)$

\begin{tabular}{llll}
\hline SNP ID & rs76999397 & rs189013166 & rs199772854 \\
\cline { 2 - 4 } rs76999397 & - & 0.81 & 0.78 \\
rs189013166 & 1 & - & 0.63 \\
rs199772854 & 1 & 1 & - \\
\hline
\end{tabular}

Table 6 Linkage disequilibrium statistics among COL6A1 SNPS $\left(D^{\prime} / R^{2}\right)$

\begin{tabular}{llll}
\hline SNP ID & rs151158105 & rs201153092 & rs13051496 \\
\hline rs151158105 & - & 0.05 & 0.07 \\
rs201153092 & 1 & - & 0.6 \\
rs13051496 & 1 & 0.91 & - \\
\hline
\end{tabular}

patients. From the current analysis, we identified five new potential pathogenic loci for T-OPLL: rs201153092 and rs13051496 in the COL6A1 gene; and rs199772854, rs76999397, and rs189013166 in the IL17RC gene. The results of the current study may be beneficial for clarifying the molecular etiology of T-OPLL.

Further genetic studies with more participants of other ethnicities are required to confirm these positive findings, and functional studies will further our understanding of this obscure disease.

\section{Abbreviations}

BMP2: Bone morphogenetic protein 2; BMP4: Bone morphogenetic protein 4; COL11A2: Collagen type XII a2 chain; COL6A1: Collagen type VI a1 chain; IL15RA: Interleukin 15 receptor subunit Q; IL17RC: Interleukin 17 receptor C; IL1B: Interleukin 1 $\beta$; OPLL: Ossification of the posterior longitudinal ligament; RUNX2: Runt-related transcription factor 2; SNPs: Single nucleotide polymorphisms; TGFB1: Transforming growth factor $\beta 1$; TLR5: Toll-like receptor 5; T-OPLL: Thoracic ossification of the posterior longitudinal ligament

\section{Acknowledgements}

Thanks are due to the Medical Research Center of Peking University Third Hospital for providing technical guidance.

Funding

This work was supported by the National Natural Science Foundation of China (81472041).

\section{Availability of data and materials}

All data generated or analyzed during this study are included in this published article.

\section{Authors' contributions}

$P W, B Z, Y M$, and $X L$ conceived of and designed the experiments. PW, YM, LY, and $\mathrm{CL}$ performed the experiments. $\mathrm{ZT}, \mathrm{YW}, \mathrm{XL}$, and $\mathrm{GH}$ analyzed the data. PW wrote the paper. $X \mathrm{~L}$ revised the manuscript. All authors reviewed the manuscript. All authors read and approved the final manuscript.

\section{Ethics approval and consent to participate}

The study protocol was approved by the Ethics Committee for Human Subjects of the Peking University Third Hospital. Informed consent was provided by all participating individuals.

\section{Consent for publication}

Not applicable

\section{Competing interests}

The authors declare that they have no competing interests.

\section{Publisher's Note}

Springer Nature remains neutral with regard to jurisdictional claims in published maps and institutional affiliations.

\section{Author details}

'Department of Orthopedics, Peking University Third Hospital, 49 North Garden Street, Haidian District, Beijing 100191, People's Republic of China. 
${ }^{2}$ The Center for Pain Medicine, Peking University Third Hospital, Beijing, People's Republic of China. ${ }^{3}$ Department of Radiology, Peking University Third Hospital, Beijing 100191, People's Republic of China.

Received: 18 August 2017 Accepted: 19 February 2018

Published online: 15 May 2018

\section{References}

1. Kawaguchi $Y$, Nakano M, Yasuda T, et al. Serum biomarkers in patients with ossification of the posterior longitudinal ligament (OPLL): inflammation in OPLL. PLoS One. 2017:12:e174881.

2. Kim KH, Kuh SU, Park JY, et al. Association between BMP-2 and COL6A1 gene polymorphisms with susceptibility to ossification of the posterior longitudinal ligament of the cervical spine in Korean patients and family members. Genet Mol Res. 2014;13:2240-7.

3. Ohtsuka K, Terayama K, Yanagihara M, et al. A radiological population study on the ossification of the posterior longitudinal ligament in the spine. Arch Orthop Trauma Surg. 1987;106:89-93.

4. Smith ZA, Buchanan CC, Raphael D, Khoo LT. Ossification of the posterior longitudinal ligament: pathogenesis, management, and current surgical approaches. Neurosurg Focus. 2011;30:E1-E10.

5. Ikegawa S. Genetics of ossification of the posterior longitudinal ligament of the spine: a mini review. J Bone Meta. 2014;21:127-32.

6. Chung W, Nam D, Jo D, Lee J. Association of toll-like receptor 5 gene polymorphism with susceptibility to ossification of the posterior longitudinal ligament of the spine in Korean population. J Korean Neurosurg Soc. 2011:49:8-12.

7. Koga H. Genetic mapping of ossification of the posterior longitudinal ligament of the spine. Hum Genet. 1998;62(6):1460-7.

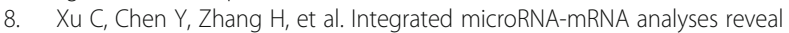
OPLL specific microRNA regulatory network using high-throughput sequencing. Sci Rep-UK. 2016;6:21580

9. Kong Q, Ma X, Li F, et al. COL6A1 polymorphisms associated with ossification of the ligamentum flavum and ossification of the posterior longitudinal ligament. Spine (Phila Pa 1976). 2007:32:2834-8.

10. Tanaka T, Ikari K, Furushima K, et al. Genomewide linkage and linkage disequilibrium analyses identify COL6A1, on chromosome 21, as the locus for ossification of the posterior longitudinal ligament of the spine. Am J Hum Genet. 2003;73:812-22.

11. Liu Y, Zhao Y, Chen Y, Shi G, Yuan W. RUNX2 polymorphisms associated with OPLL and OLF in the Han population. Clin Orthop Relat Res. 2010;468:3333-41.

12. Wang H, Liu D, Yang Z, et al. Association of bone morphogenetic protein-2 gene polymorphisms with susceptibility to ossification of the posterior longitudinal ligament of the spine and its severity in Chinese patients. Eur Spine J. 2008:17:956-64.

13. Furushima K, Shimo-Onoda K, Maeda S, et al. Large-scale screening for candidate genes of ossification of the posterior longitudinal ligament of the spine. J Bone Miner Res. 2002;17:128-37.

14. Kamiya M. Association between a polymorphism of the transforming growth factor- $\beta 1$ gene and genetic susceptibility to ossification of the posterior longitudinal ligament in Japanese patients. Spine. 2001;2:1264-7.

15. Ogata N, Koshizuka Y, Miura T, et al. Association of bone metabolism regulatory factor gene polymorphisms with susceptibility to ossification of the posterior longitudinal ligament of the spine and its severity. Spine (Phila Pa 1976). 2002;27:1765-71.

16. Guo Q, Lv SZ, Wu SW, Tian X, Li ZY. Association between single nucleotide polymorphism of IL15RA gene with susceptibility to ossification of the posterior longitudinal ligament of the spine. J Orthop Surg Res. 2014:9:103.

17. IKEGAWA S. Genomic study of ossification of the posterior longitudinal ligament of the spine. Proc Jpn Acad Ser B Phys Biol Sci. 2014;90:405-12.

18. $\operatorname{Sim} \mathrm{NL}$, Kumar P, Hu J, Henikoff S, Schneider G, Ng PC. SIFT web server: predicting effects of amino acid substitutions on proteins. Nucleic Acids Res. 2012:40:W452-7.

19. Adzhubei IA, Schmidt S, Peshkin L, et al. A method and server for predicting damaging missense mutations. Nat Methods. 2010;7:248-9.

20. Schwarz JM, Cooper DN, Schuelke M, Seelow D. MutationTaster2: mutation prediction for the deep-sequencing age. Nat Methods. 2014;11:361-2.

21. Davydov EV, Goode DL, Sirota M, Cooper GM, Sidow A, Batzoglou S. Identifying a high fraction of the human genome to be under selective constraint using GERP++. PLoS Comput Biol. 2010;6:e1001025.
22. Tsuyama N. Ossification of the posterior longitudinal ligament of the spine. Clin Orthop Relat Res. 1984;71-84

23. Gaudet M, Fara AG, Beritognolo I, Sabatti M. Allele-specific PCR in SNP genotyping. Methods Mol Biol. 2009:578:415-24.

24. Kryukov GV, Pennacchio LA, Sunyaev SR. Most rare missense alleles are deleterious in humans: implications for complex disease and association studies. Am J Hum Genet. 2007:80:727-39.

25. Wiberg C, Klatt AR, Wagener R, et al. Complexes of matrilin-1 and biglycan or decorin connect collagen VI microfibrils to both collagen II and aggrecan. J Biol Chem. 2003;278:37698-704.

26. Tsukahara S, Miyazawa N, Akagawa H, et al. COL6A1, the candidate gene for ossification of the posterior longitudinal ligament, is associated with diffuse idiopathic skeletal hyperostosis in Japanese. Spine (Phila Pa 1976). 2005;30:2321-4.

27. Ho AW, Gaffen SL. IL-17RC: a partner in IL-17 signaling and beyond. Semin Immunopathol. 2010;32:33-42.

28. Huang $\mathrm{H}, \mathrm{Kim} \mathrm{HJ}$, Chang EJ, et al. IL-17 stimulates the proliferation and differentiation of human mesenchymal stem cells: implications for bone remodeling. Cell Death Differ. 2009;16:1332-43.

29. Han IB, Ropper $A E$, Jeon $Y J$, et al. Association of transforming growth factor-beta 1 gene polymorphism with genetic susceptibility to ossification of the posterior longitudinal ligament in Korean patients. Genet Mol Res. 2013;12:4807-16.

\section{Submit your next manuscript to BioMed Central and we will help you at every step:}

- We accept pre-submission inquiries

- Our selector tool helps you to find the most relevant journal

- We provide round the clock customer support

- Convenient online submission

- Thorough peer review

- Inclusion in PubMed and all major indexing services

- Maximum visibility for your research

Submit your manuscript at www.biomedcentral.com/submit
Biomed Central 